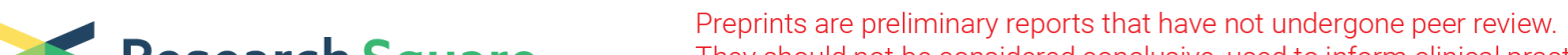 Research Square They should not be considered conclusive, used to inform clinical practice, or referenced by the media as validated information.
}

\section{The Short-Term Efficacy of Novel No-Touch Combined Directional Perfusion Radiofrequency Ablation in the Treatment of Small Hepatocellular Carcinoma With Cirrhosis.}

\section{Chengming Qu}

Third Military Medical University: Army Medical University https://orcid.org/0000-0002-9773-8815

\section{Xin-Qian Li}

Renmin Hospital of Wuhan University: Wuhan University Renmin Hospital

\section{Feng Xia}

Third Military Medical University: Army Medical University

Kai Feng ( $\sim$ fengkai7688@hotmail.com)

Third Military Medical University (Army Medical University) https://orcid.org/0000-0001-6027-8002

\section{Kuansheng Ma}

Third Military Medical University: Army Medical University

\section{Research article}

Keywords: radiofrequency ablation, no-touch, directional perfusion, hepatocellular carcinoma, minimally invasive treatment

Posted Date: November 20th, 2020

DOl: https://doi.org/10.21203/rs.3.rs-111374/v1

License: (c) (i) This work is licensed under a Creative Commons Attribution 4.0 International License. Read Full License

Version of Record: A version of this preprint was published at Journal of Investigative Surgery on June 4th, 2021. See the published version at https://doi.org/10.1080/08941939.2021.1931575. 


\section{Abstract}

\section{Background}

No-touch combined directed perfusion radiofrequency ablation (NTDP-RFA) is a new technique for the treatment of hepatocellular carcinoma (HCC). The purpose of this study was to evaluate the short-term efficacy of this new technique for the treatment of small HCC with cirrhosis.

\section{Methods}

From January 2017 to March 2018, 56 consecutive patients treated with NTDP-RFA at our center were enrolled in this retrospective study. All NTDP-RFA procedures involved the use of internally cooled wet electrodes with a directional injection function, which can perform both intraelectrode cooling and extraelectrode saline perfusion. Survival curves were analyzed using Kaplan-Meier methods, and Cox proportional hazards regression analyses were used to assess predictors of tumor progression and overall survival. Operative characteristics and complications were also assessed.

Results

No technical failure occurred, and the complete ablation rate after single NTDP-RFA treatment was $98.2 \%$. The median ablation time was only 8 (6-8) min. Only 5 patients (8.9\%) experienced mild complications postoperation, and the median hospital stay was only 4 (3-5) days. In the 18 patients (32.1\%) with poor liver function reserve (indocyanine green retention rate at $15 \mathrm{~min}>15 \%$ ), their liver function returned to normal on the third day after the postoperation. The 1-year and 2-year local and distant progression rates were $1.7 \%, 7.1 \%, 3.5 \%$ and $10.7 \%$, respectively.

\section{Conclusions}

NTDP-RFA in the treatment of small HCC with cirrhosis has a low incidence of complications and provides a high survival rate without local tumor progression. Further prospective randomized controlled studies are needed to investigate the long-term results.

\section{Background}

Liver cancer is the fourth leading cause of cancer-related deaths globally ${ }^{1}$. Hepatocellular carcinoma (HCC) accounts for $70 \%$ to $85 \%$ of all primary HCCs and is the main cause of death in patients with cirrhosis $^{2,3}$. In recent years, due to the increasing awareness of physical examinations and continuous improvements in medical monitoring programs for chronic hepatitis and cirrhosis, an increasing number of patients have been diagnosed with small $\mathrm{HCC}^{4}(\leq 3 \mathrm{~cm})$. Radical therapies ${ }^{5}$ for small HCC include hepatectomy, liver transplantation, and percutaneous thermal ablation (e.g., microwave ablation and radiofrequency ablation, RFA). RFA has been widely used in the treatment of small HCC worldwide due to its low invasiveness, safety, economy and effectiveness ${ }^{6}$. 
Conventional intratumor monopolar RFA can produce only limited necrotic areas, and even if overlapping ablation is performed, it will lead to uneven necrosis ${ }^{7}$. Therefore, local tumor progression (LTP) is often caused by untreated satellite nodules ${ }^{8}$ or incomplete ablation ${ }^{9}$. No-touch multipolar RFA (NTM-RFA) represents a new type of local ablation surgery. Due to its increased ablation volume and significantly reduced risk of tumor spread along the needle tract, it has become the first-line treatment for small HCC in many medical institutions ${ }^{10,11}$. However, for patients with HCC with a background of cirrhosis (even small $\mathrm{HCC})^{12}$, chronic inflammation and cirrhosis can cause liver insufficiency, which also limits the amount of tolerable ablation of the liver parenchyma and increases the risk of liver failure and death after NTM-RFA. Therefore, on this basis, we developed a new type of a no-touch combined directional perfusion RFA (NTDP-RFA) system. By inserting two or more electrodes around the tumor and activating them at the same time, hypertonic saline can be directed into the lesion from the side hole through the tube in the electrode to produce more uniform and more thorough necrosis. While increasing the maximum ablation volume, this technique also reduces the damage to normal tissues in the direction of nonperfusion, thereby reducing the incidence of postoperative complications. Therefore, the purpose of this study was to evaluate the short-term efficacy of NTDP-RFA in the treatment of small HCC with cirrhosis.

\section{Methods}

\section{Patients}

The medical records of patients with small HCC who received NTDP-RFA at our center between January 2017 and March 2018 were retrieved and retrospectively analyzed (Fig. 1). A total of 56 consecutive patients were included in this retrospective study.

The inclusion criteria for this study were as follows: (1) 18-70 years old; (2) primary HCC with a background of cirrhosis; (3) single nodules with a diameter $\leq 3 \mathrm{~cm}$; (4) no invasion of the portal vein, the hepatic vein trunk or secondary branches; (5) no intrahepatic and extrahepatic metastases; (6) Child-Pugh grade A/B; (7) the indocyanine green retention rate at $15 \mathrm{~min}$ (ICGR-15) $\leq 30 \%$; (8) follow-up period of up to 2 years; and (9) no other antitumor therapy received before treatment. The exclusion criteria for this study were as follows: (1) lost-to-follow-up or follow-up period of less than 2 years; (2) multiple HCC nodules or a single HCC nodule $>3 \mathrm{~cm}$; (3) patients with severe portal hypertension, a history of esophageal variceal hemorrhage, severe hypersplenism syndrome, or refractory ascites; (4) lesions adjacent to the gallbladder, important blood vessels and bile ducts of the hepatic hilum and surrounding organs; (5) patients who were willing to receive hepatectomy or liver transplantation; and (6) severe bleeding tendency, platelet count $<50 \times 10^{\wedge} 9 / \mathrm{L}$, or prolonged prothrombin time $>3$ seconds.

\section{Diagnosis of HCC and cirrhosis}

Before NTDP-RFA treatment, all patients underwent imaging studies, including contrast-enhanced computed tomography (CT) and/or magnetic resonance imaging (MRI). To plan the NTDP-RFA procedure, 
all patients underwent contrast-enhanced ultrasound (US) before NTDP-RFA. he diagnosis of HCC and cirrhosis was confirmed by biopsy during the NTDP-RFA procedure.

\section{Equipment: RF electrodes and RF systems}

All NTDP-RFA procedures involved the use of internally cooled wet electrodes with a directional injection function (LDDJS1-0200200, Mianyang Lide Electronics Co., Ltd., Mianyang, China), which can perform both intraelectrode cooling and extraelectrode saline perfusion. The electrode has an outer diameter of 14 $\mathrm{G}$, and there is a pipe inside to circulate the cooled hypertonic saline in the electrode, and the perfusion rate is set to $40 \mathrm{ml} / \mathrm{min}$. The tip of the electrode has an exposed hot end with a length of $2 \mathrm{~cm}$. At distance of $0.5 \mathrm{~cm}$ and $1.5 \mathrm{~cm}$ from the tip, there are only two injection holes on one side of the hot end, each of which is $0.5 \mathrm{~cm}$ long and $0.2 \mathrm{~cm}$ wide. A solution of $5 \% \mathrm{NaCl}$ was used as the infusion solution, and the flow rate was adjusted to $1 \mathrm{ml} / \mathrm{min}$. The RF energy is provided by a $400 \mathrm{kHz}$ RF generator (LDRF120S, Mianyang Lide Electronics Co., Ltd., Mianyang, China), which can activate multiple electrodes simultaneously.

\section{Preoperative preparation}

All RFA procedures were performed by a hepatobiliary surgeon with more than 15 years of experience in RFA. All operations were performed under monitoring anesthesia, and puncture was performed percutaneously under the guidance of real-time US. An injection of fentanyl citrate (0.1-0.2 mg, Humanwell Pharmaceutical Co., Ltd., Yichang, China) was used for analgesia, an injection of dexmedetomidine hydrochloride (50-100 mg, Hengrui Medicine Co., Ltd., Jiangsu, China) was administered for sedation, and an injection of lidocaine hydrochloride (Zhaohui Pharmaceutical Co., Ltd., Shanghai, China) was used for topical anesthesia. The number of RF electrodes used depended on the diameter of the tumor. For tumors with a diameter of $\leq 20 \mathrm{~mm}$, two electrodes were used; for tumors with a diameter between 20-30 mm, three electrodes were used. Preoperative contrast-enhanced US was performed to identify the tumor site. The optimal treatment position and needle insertion route (selected to avoid large blood vessels, bile ducts and adjacent organs) were selected, and the puncture point was marked on the skin.

\section{NTDP-RFA procedure}

The specific implementation steps of NTDP-RFA were as follows: two or three electrodes were inserted in parallel into the liver parenchyma around the target tumor, and the side hole at the hot end of the electrodes was pointed in the direction of the tumor. The precise placement of the electrodes is shown in Fig. 2. The initial power was set to $60 \mathrm{~W}$, and then the power was increased at a rate of $10 \mathrm{~W} / \mathrm{min}$ until the maximum power of $120 \mathrm{~W}$ was reached. During ablation, hypertonic saline solution was continuously injected into the tissue through the side hole of the electrode, and the RFA system continuously measured tissue resistance. The power output was stopped automatically if resistance exceeded a specific limit $(300 \Omega)$. Therefore, the time of the procedure changed according to the situation. At the same time, the electrode interior was cooled by the circulating perfusion of saline during ablation. The ablation 
procedures were monitored by real-time US, and the ablation was not terminated until the transient hyperechoic cloud caused by the gas covered all units of the tumor. After ablation was completed, the electrode needle was removed in "needle tract ablation mode $(35 \mathrm{~W})$ " to prevent bleeding.

\section{Follow-up and definitions of terminology}

At 15 min after NTDP-RFA completion, contrast-enhanced US was immediately reexamined to evaluate the therapeutic effect. Complete ablation was defined as no enhancement area during the arterial and portal phases. If there were discontinuous, focal or nodular enhancement areas at the tumor site, indicating incomplete treatment, NTDP-RFA was performed again. In the first and second months after NTDP-RFA and every two months thereafter, patients were followed up by contrast-enhanced US, CT or MRI. LTP was defined as the appearance of new tumor foci within $2 \mathrm{~cm}$ of the edge of the ablation area by at least one follow-up imaging examination. Otherwise, new tumors were defined as distant tumor progression (classified as intrahepatic distant recurrence and extrahepatic metastasis). Intrahepatic distant recurrence was defined as the detection of a tumor lesion $2 \mathrm{~cm}$ away from the ablation position within the liver. A visual analog scale (VAS) was used to assess postoperative pain on a scale of 0 to 10 ( 0 , no pain; 10 , worst imaginable pain). Postoperative complications were also assessed according to the Society of Interventional Radiology (SIR) classification. Technical success was defined as completion of the NTDP-RFA procedure as planned. Fifteen minutes after completion of the NTDP-RFA procedure, contrast-enhanced US indicated that complete ablation was defined as clinical effectiveness.

\section{Study endpoints}

The primary endpoint of the study was the rate of LTP. The secondary endpoints were the comparison of (i) intrahepatic distant recurrence rates; (ii) tumor progression-free survival rates; and (iii) overall survival rates. Tumor progression-free survival and overall survival were calculated from the date of initial NTDPRFA treatment to the date of tumor recurrence or death.

\section{Statistical analyses}

Continuous data are expressed as medians and interquartile ranges. Categoric variables are expressed as frequencies and proportions. Repeated measures ANOVA was used to compare biochemical indicators of liver function. Survival curves were estimated by using the Kaplan-Meier method. Cox proportional hazards regression analyses were performed to determine significant baseline clinical-biologic and nodule parameters for the prediction of local and overall tumor progression-free survival rates. All variables with $P<0.200$ were included in the multivariate analysis; a stepwise Cox proportional hazards regression model was used to evaluate their value as independent predictors. In stepwise regression, $P<$ 0.05 was considered to indicate a significant difference. SPSS for version 25.0 (IBM, Armonk, New York, USA) was used for statistical analysis.

\section{Results}




\section{Patient characteristics}

The baseline characteristics of the patients are summarized in Table 1. Fifty-six patients had primary HCC: 49 males (87.5\%) and 7 females (12.5\%). The median patient age was 55 (46-63) years. Thirty-nine patients (69.7\%) had hepatitis B cirrhosis, 18 (32.1\%) had an ICGR-15> 15\%, and 8 (14.2\%) had a serum AFP level greater than $200 \mathrm{ng} / \mathrm{mL}$. The median tumor diameter was 26 (18.0-28.0) mm: 19 patients had a diameter $\leq 20 \mathrm{~mm}$, and 37 patients had a diameter $>20 \mathrm{~mm}$ and $\leq 30 \mathrm{~mm}$.

\section{Operative characteristics and complications}

The operative characteristics of the patients are shown in Table 2. All patients completed the NTDP-RFA procedure as planned. The median duration of NTDP-RFA was 8 (6-8) min. The length of hospital stay was calculated from the first day after NTDP-RFA, and the median length of hospital stay was 4 (3-5) days. Fifteen minutes after the completion of NTDP-RFA, contrast-enhanced US indicated that the median ablation diameter was 49.0 (44.2-51.0) $\mathrm{mm}$. Complete ablation was achieved in 55 patients after one NTDP-RFA treatment and in 1 patient after two NTDP-RFA treatments. No treatment-related deaths occurred in this study. Postoperative grade A complications of pleural effusion occurred in 5 patients (8.9\%), including 2 patients with mild ascites. All patients returned to normal within 2-4 days after conservative or symptomatic treatment.

As shown in Fig. 3, the patients were divided into two groups according to preoperative ICGR-15, and the perioperative liver function indexes of the patients were analyzed and compared. When comparing the two groups,the Tbil, ALT and AST levels in the ICGR-15 > 15\% group were significantly higher than those in the ICGR-15 $\leq 15 \%$ group on the first postoperative day $(P<0.050)$. Compared with the preoperative levels, the postoperative Tbil, ALT and AST levels were significantly higher in both groups on day 1 after NTDP-RFA treatment $(P<0.001)$ and significantly lower on day 3 after liver function protection $(P<$ 0.010) (Fig. $3 a, b, c)$. In addition, the Alb level was significantly lower on the first day after NTDP-RFA than preoperation $(P<0.050)$, and no difference was found on postoperative day 3 (Fig. 3 e). The ALP and PT-INR levels were compared, but no differences were found between the two groups at any postoperative time compared with the preoperative time (Fig. $3 d, f$ ).

\section{Local and distant tumor progression and survival}

During the follow-up period, 4 patients (7.1\%) developed local recurrence at 12, 18, 20, and 24 months after NTDP-RFA treatment. Among them, only 1 patient with a tumor diameter $\leq 20 \mathrm{~mm}$ developed LTP at the 24th month after NTDP-RFA treatment. Univariate analysis did not reveal characteristics associated with LTP. The 1-year and 2-year local progression-free survival rates were 98\% (95\% Cl: 94\%, 99\%) and 92\% (95\% Cl: 85\%, 98\%), respectively (Fig. 4). The distant tumor progression (intrahepatic distant recurrence and extrahepatic metastasis) rates at 1 and 2 years after NTDP-RFA treatment were $3.5 \%$ and $10.7 \%$, respectively. Two of the patients had distant solid organ metastases (one in the lung and one in the bone), with tumor diameters $>20 \mathrm{~mm}$. 


\section{Overall tumor progression and survival}

Table 3 shows the predictors of the 2 -year overall tumor recurrence rate. Age $\geq 60$ years, ICGR-15 > 15\%, prothrombin activity $<75 \%$, platelet count $<100 \mathrm{~g} / \mathrm{L}$, serum total bilirubin $>21 \mu \mathrm{mol} / \mathrm{L}$, serum AFP $>200$ $\mathrm{ng} / \mathrm{mL}$ and tumor diameter $>20 \mathrm{~mm}$ and $\leq 30 \mathrm{~mm}$ were used as references. In the univariate analysis, ICGR-15 $>15 \%(P=0.16$, hazard ratio $=2.41[95 \% \mathrm{Cl}: 0.69,8.33])$ and diameter $>20 \mathrm{~mm}$ and $\leq 30 \mathrm{~mm}(P$ $=0.11$, hazard ratio $=5.26$ [95\% Cl: $0.66,41.58]$ ) were associated with overall tumor recurrence. However, when included in the multivariate analysis, there were no characteristics associated with the overall recurrence rate. The 1-year and 2-year progression-free survival rates were 95\% (95\% Cl: 89\%, 99\%) and $82 \%$ (95\% Cl: $73 \%, 92 \%)$, respectively.

\section{Management of patients who experienced recurrence}

Of the 4 patients with LTP, 3 received NTDP-RFA treatment again, and one received liver transplantation. Two of the 4 patients with intrahepatic distant recurrence received surgical resection, and the other two received NTDP-RFA again.

\section{Discussion}

Over the past two decades, RFA has become the most widely used local ablative technique for liver cancer due to its minimally invasive nature, safety, effectiveness and repeatability ${ }^{13-15}$. However, in the traditional unipolar RFA mode, a single electrode is placed directly in the center of the target tumor, and the RF current is centrifugally diffused from the center to the surrounding area. This can easily lead to tumor spread along the needle path and vascular proliferation ${ }^{16}$. Compared with surgical resection, the higher incidence of postoperative LTP is also one of the main limitations of unipolar RFA ${ }^{17}$. For tumors with a diameter of more than $20 \mathrm{~mm}$, complete ablation and an ablation boundary greater than $10 \mathrm{~mm}$ are required, often with overlapping ablation ${ }^{18-20}$. However, the hyperechoic cloud generated after RFA will cover the treatment area, so it is a difficult challenge to perform overlapping ablation under the guidance of US. Therefore, there is a risk of incomplete ablation and residual satellite lesions, which will lead to LTP21. To overcome the limitations of unipolar RFA and improve the clinical effectiveness of RFA in the treatment of HCC, NTM-RFA has been applied in clinical practice in recent years ${ }^{22}$.

In the NTM-RFA mode, the electrodes are placed around the lesion, and the RF current flows between two or more electrodes, that is, the current diffuses centripetally from the periphery to the center. This enables the RF current to be concentrated between the tips of the electrodes, making it easier to deposit highdensity currents into target tissues, including tumors, thus achieving a larger ablation area ${ }^{23}$. Moreover, the operation does not directly touch the tumor, nor does it increase the pressure within the tumor, and the risks of tumor spread across blood vessels and implantation and metastasis are also greatly reduced $^{24,25}$. However, the disadvantage of the NTM-RFA mode is that the temperature in the RF current concentration area is too high in a short time, which will lead to rapid carbonization of the tissue (especially the tissue directly contacting the metal electrode) and the rapid increase in the impedance, so 
that the RF energy transfer is insufficient. The carbonized tissue will also adhere to the tip of the electrode, resulting in uneven transmission of the RF current, thus reducing the heat production efficiency of unit RF energy. Moreover, with the increase in tumor volume, the tumor far from the electrode may not reach the critical temperature for complete necrosis, which may lead to LTP during follow-up. Therefore, to achieve a larger ablation area, it is necessary to increase the power or prolong the operation time, resulting in a larger ablation area outside the target tumor. However, this will not only increase the risk of thermal injury to the surrounding organs but also prolong the postoperative recovery time, especially for patients with $\mathrm{HCC}$ with cirrhosis who have different degrees of liver function damage.

When RFA is used to treat HCC with cirrhosis with poor liver function reserve, to achieve a balance between the ablation area and liver function, we believe that the most ideal situation is to completely ablate the tumor and reach the ablation boundary of more than $10 \mathrm{~mm}$ and can effectively avoid unnecessary damage to the liver parenchyma and the risk of any disseminated tumor. Therefore, we developed an NTDP-RFA system with the aims of creating an ablation area at least $10 \mathrm{~mm}$ beyond the tumor area in three dimensions and obtaining complete tumor necrosis. During RFA treatment, hypertonic saline can be injected into the target lesion from the side hole through the tube, which not only reduces the carbonization of tissue in the current concentration area and slows the speed of impedance increase but also increases the electrical conductivity and thermal conductivity ${ }^{26,27}$. Thus, without blindly increasing the power and prolonging the operation time, greater RF energy deposition will be caused, and the ablation area will be significantly expanded ${ }^{28}$. Furthermore, when hypertonic saline is injected into the lesion tissue directionally and evenly, it cannot only produce regular and uniform necrosis but also reduce damage to normal tissues in other directions and retain the noncancerous part of the liver parenchyma to the maximum extent. Studies ${ }^{29}$ have also shown that activating multiple wet electrodes at the same time can significantly reduce the application time. Moreover, the system combined with adaptive pulse technology can adjust the output of RF energy according to the change in lesion impedance so that ablation will not be affected by the "heat sink effect", thus achieving the expected ablation range. Finally, a sufficient ablation boundary and complete tumor necrosis can be generated to reduce the incidence of LTP after NTDP-RFA. This also broadens the application prospect of precision medicine in minimally invasive treatment. To our knowledge, there is no report on the application of this new technology in the treatment of small HCC with the background of cirrhosis.

In our study, the median HCC diameter was 26 (18.0-28.0) $\mathrm{mm}$, the median ablation time was only 8 (6-8) min, and the median ablation area diameter was 49.0 (44.2-51.0) $\mathrm{mm}$, which was more than $10 \mathrm{~mm}$ beyond the tumor margin. The clinical efficiency was up to $98.2 \%(55 / 56)$, and the technical success rate was $100 \%$. Even for 18 patients (32.1\%) with poor liver function reserve (ICGR-15 > 15\%), their liver function returned to normal on the third day postoperation. The median postoperative hospital stay was only 4 (3-5) days. After NTDP-RFA treatment, only 5 patients (8.9\%) experienced grade A complications. No patient needed surgical intervention, and all patients recovered completely. The 1-year and 2-year local and distant progression rates were $1.7 \%, 7.1 \%, 3.5 \%$ and $10.7 \%$, respectively. Lencioni ${ }^{30}$ reported that after unipolar RFA treatment for tumors with a median diameter of $28 \mathrm{~mm}$ and a median follow-up time 
of 24 months, the LTP rate reached 10\%. Kayvan Mohkam et al. ${ }^{31}$ reported that after NTM-RFA treatment of HCC with a median diameter of 30 (25-40) mm, the 1-year and 3-year local and distant tumor progression rates reached $5.5 \%, 10.0 \%, 7.4 \%$ and $27.8 \%$, respectively, and the total incidence of complications was as high as $50 \%$, including $5.6 \%$ severe complications. Arnaud Hocquelet ${ }^{32}$ also reported NTM-RFA in the treatment of HCC patients with an average diameter of $25 \mathrm{~mm}$ (SD: 8). According to the SIR classification, the incidence of postoperative complications of grade $\mathrm{C}$ and above was as high as $7.2 \%$. The incidence of complications higher than Clavien-Dindo Grade 3A (requiring surgery, intensive care unit or death) was $4.4 \%$. One patient died of postoperative liver failure. Therefore, the incidence and grade of postoperative complications of NTDP-RFA are lower than those reported above, and the effect of local tumor control is significant.

Our study has some limitations. First, as a retrospective uncontrolled study, prospective randomized controlled trials are also needed to verify the results. In addition, a short follow-up time may underestimate the possibility of LTP. Finally, a $5 \% \mathrm{NaCl}$ solution was used as the perfusion solution, and the flow rate was adjusted to $1 \mathrm{ml} / \mathrm{min}$. Further studies are necessary to optimize the concentration and quantity of the hypertonic saline solution.

\section{Conclusions}

In conclusion, the new type of NTDP-RFA in the treatment of small HCC with cirrhosis has a low incidence of complications and provides a high survival rate without LTP. Therefore, it is a safe and effective treatment method worthy of consideration. Further prospective randomized controlled studies are needed to investigate the long-term results.

\section{Abbreviations}

NTDP-RFA: No-touch combined directed perfusion radiofrequency ablation; HCC: hepatocellular carcinoma; NTM-RFA: No-touch multipolar RFA; CT: computed tomography; MRI: magnetic resonance imaging; US: ultrasound; LTP: local tumor progression; VAS: visual analog scale; SIR: Society of Interventional Radiology; BMI: body mass index; ALT: alanine transaminase; AST: aspartate aminotransferase; ALP: alkaline phosphatase; ALB: albumin; PT-INR: prothrombin time international normalised ratio; ICGR-15: indocyanine green retention rate at 15 min; AFP: alpha-fetoprotein; HR, hazard ratio.

\section{Declarations}

\section{Ethics approval and consent to participate[}

This study was reviewed and approved by the Institutional Review Board of the Southwest Hospital of Third Military Medical University (Army Medical University), and the requirement for written informed consent was waived. All procedures were conducted in accordance with the Declaration of Helsinki. 
Consent for publication: not applicable.

Availability of data and materials: not applicable.

Competing interests: No potential conflict of interest was reported by the authors.

\section{Funding:}

This work was funded by National Natural Science Foundation of China [No. 81672857], The Chongqing Technology Innovation and Application Development Project [No. cstc2019jscx-msxmX0230], Clinical Research Fund of the Third Military Medical University [No. SWH2017ZDCX4101, No. SWH2017YBXM26].

\section{Authors' contributions:}

QCM analysed data and drafted manuscript. LXQ helped with English language editing. XF performed analysis and interpretation of all data. FK and MKS designed and supervised the study and are the cocorresponding authors. All authors approved the final version of the article to be published.

Acknowledgements: The authors thank all the medical staff at the Clinical Research Center of Southwest Hospital.

\section{References}

1. Gao Q, Zhu H, Dong L, et al. Integrated Proteogenomic Characterization of HBV-Related Hepatocellular Carcinoma. C 2019;179:1240.

2. Wang $Y, H e L, D u Y$, et al. The long noncoding RNA IncTCF7 promotes self-renewal of human liver cancer stem cells through activation of Wnt signaling. Cell Stem Cell. 2015;16:413-425.

3. Moeini A, Torrecilla S, Tovar V, et al. An Immune Gene Expression Signature Associated With Development of Human Hepatocellular Carcinoma Identifies Mice That Respond to Chemopreventive Agents. G 2019;157:1383-1397.

4. EASL-EORTC clinical practice guidelines: management of hepatocellular carcinoma. J HEPATOL 2012;56:908-943.

5. Llovet JM, Bruix J. Novel advancements in the management of hepatocellular carcinoma in 2008. J H 2008;48;Suppl 1:S20-S37.

6. Feng K, Yan J, Li X, et al. A randomized controlled trial of radiofrequency ablation and surgical resection in the treatment of small hepatocellular carcinoma. J Hepatol. 2012;57:794-802.

7. Hocquelet A, Aube C, Rode A, et al. Comparison of no-touch multi-bipolar vs. monopolar radiofrequency ablation for small HCC. J H 2017;66:67-74.

8. 8..Shi M, Guo RP, Lin XJ, et al. Partial hepatectomy with wide versus narrow resection margin for solitary hepatocellular carcinoma: a prospective randomized trial. Ann S 2007;245:36-43. 
9. Ahmed M, Solbiati L, Brace CL, et al. Image-guided tumor ablation: standardization of terminology and reporting criteria-a 10-year update. R 2014;273:241-260.

10. Seror O, N'Kontchou G, Nault JC, et al. Hepatocellular Carcinoma within Milan Criteria: No-Touch Multibipolar Radiofrequency Ablation for Treatment-Long-term Results. R 2016;280:981.

11. Kawamura Y, Ikeda K, Fujiyama S, et al. Potential of a no-touch pincer ablation procedure that uses a multipolar radiofrequency ablation system to prevent intrasubsegmental recurrence of small and single hepatocellular carcinomas. Hepatol R 2017;47:1008-1020.

12. Raut CP, Izzo F, Marra P, et al. Significant long-term survival after radiofrequency ablation of unresectable hepatocellular carcinoma in patients with cirrhosis. Ann Surg 0 2005;12:616-628.

13. Chen MS, Li JQ, Zheng Y, et al. A prospective randomized trial comparing percutaneous local ablative therapy and partial hepatectomy for small hepatocellular carcinoma. Ann S 2006;243:321-328.

14. Lau WY, Leung TW, Yu SC, Ho SK. Percutaneous local ablative therapy for hepatocellular carcinoma: a review and look into the future. Ann S 2003;237:171-179.

15. Wang JH, Wang $\mathrm{CC}$, Hung $\mathrm{CH}$, Chen $\mathrm{CL}$, Lu SN. Survival comparison between surgical resection and radiofrequency ablation for patients in BCLC very early/early stage hepatocellular carcinoma. $\mathrm{J} \mathrm{H}$ 2012;56:412-418.

16. Snoeren N, Huiskens J, Rijken AM, et al. Viable tumor tissue adherent to needle applicators after local ablation: a risk factor for local tumor progression. Ann Surg 0 2011;18:3702-3710.

17. Hasegawa K, Makuuchi M, Takayama T, et al. Surgical resection vs. percutaneous ablation for hepatocellular carcinoma: a preliminary report of the Japanese nationwide survey. $\mathrm{J} \mathrm{H} \mathrm{2008;49:589-}$ 594.

18. Chen MH, Yang W, Yan K, et al. Large liver tumors: protocol for radiofrequency ablation and its clinical application in 110 patients-mathematic model, overlapping mode, and electrode placement process. R 2004;232:260-271.

19. Lee JM, Han JK, Kim HC, et al. Switching monopolar radiofrequency ablation technique using multiple, internally cooled electrodes and a multichannel generator: ex vivo and in vivo pilot study. Invest R 2007;42:163-171.

20. Hansler J, Frieser M, Tietz V, et al. Percutaneous ultrasound-guided radiofrequency ablation (RFA) using saline-perfused (wet) needle electrodes for the treatment of hepatocellular carcinoma-long term experience. Ultraschall M 2007;28:604-611.

21. Dodd GR, Frank MS, Aribandi M, Chopra S, Chintapalli KN. Radiofrequency thermal ablation: computer analysis of the size of the thermal injury created by overlapping ablations. Am J Roentgenol. 2001;177:777-782.

22. Kawamura Y, Ikeda K, Shindoh J, et al. No-touch ablation in hepatocellular carcinoma has the potential to prevent intrasubsegmental recurrence to the same degree as surgical resection. Hepatol R 2019;49:164-176.

23. Lee JM, Han JK, Kim SH, et al. A comparative experimental study of the in-vitro efficiency of hypertonic saline-enhanced hepatic bipolar and monopolar radiofrequency ablation. Korean J R 
2003;4:163-169.

24. Kang TW, Lim HK, Lee MW, et al. Aggressive Intrasegmental Recurrence of Hepatocellular Carcinoma after Radiofrequency Ablation: Risk Factors and Clinical Significance. R 2015;276:274-285.

25. Hocquelet A, Balageas P, Frulio N, Trillaud H. Aggressive Intrasegmental Recurrence of Periportal Hepatocellular Carcinoma after Radiofrequency Ablation: Role of Ablative Technique and Heat-Sink Effect? R 2015;276:932-933.

26. Goldberg SN, Ahmed M, Gazelle GS, et al. Radio-frequency thermal ablation with $\mathrm{NaCl}$ solution injection: effect of electrical conductivity on tissue heating and coagulation-phantom and porcine liver study. R 2001;219:157-165.

27. Burdio F, Guemes A, Burdio JM, et al. Hepatic lesion ablation with bipolar saline-enhanced radiofrequency in the audible spectrum. Acad R 1999;6:680-686.

28. Lee JM, Han JK, Kim SH, et al. Saline-enhanced hepatic radiofrequency ablation using a perfusedcooled electrode: comparison of dual probe bipolar mode with monopolar and single probe bipolar modes. Korean J R 2004;5:121-127.

29. Hansler J, Frieser M, Tietz V, et al. Percutaneous ultrasound-guided radiofrequency ablation (RFA) using saline-perfused (wet) needle electrodes for the treatment of hepatocellular carcinoma-long term experience. Ultraschall Med. 2007;28:604-611.

30. Lencioni RA, Allgaier HP, Cioni D, et al. Small hepatocellular carcinoma in cirrhosis: randomized comparison of radio-frequency thermal ablation versus percutaneous ethanol injection. $R$ 2003;228:235-240.

31. Mohkam K, Dumont $P$, Manichon A, et al. No-touch multibipolar radiofrequency ablation vs. surgical resection for solitary hepatocellular carcinoma ranging from 2 to $5 \mathrm{~J}$ Hepatol. 2018;68:1172-1180.

32. Hocquelet $A$, Aubé $C$, Rode $A$, et al. Comparison of no-touch multi-bipolar vs. monopolar radiofrequency ablation for small HCC. J H 2017;66:67-74.

\section{Tables}

Table 1. Characteristics of 56 patients treated with NTDP-RFA. 


\begin{tabular}{|c|c|}
\hline Variables & Date \\
\hline Age, years & $55(46-63)$ \\
\hline Sex, male & $49(87.5)$ \\
\hline $\mathrm{BMI}, \mathrm{kg} / \mathrm{m}^{2}$ & $23.8(22.2-25.3)$ \\
\hline \multicolumn{2}{|l|}{ Cirrhosis aetiologies } \\
\hline Alcohol-related & $11(19.6)$ \\
\hline Hepatitis B & $39(69.7)$ \\
\hline Hepatitis C & $6(10.7)$ \\
\hline \multicolumn{2}{|l|}{ Laboratory data } \\
\hline ALT, IU/L & $35.7(27.9-54.9)$ \\
\hline AST, IU/L & $31.6(27.2-38.1)$ \\
\hline ALP, IU/L & $73(64.0-83.2)$ \\
\hline ALB, $g / L$ & $41.9(38.1-45.7)$ \\
\hline PT-INR & $0.91(0.86-0.96)$ \\
\hline ICGR-15, \% & $7.8(5.9-15.6)$ \\
\hline PTA (prothrombin activity), \% & $76.6(66.9-81.6)$ \\
\hline Platelet count, $10^{9} / \mathrm{L}$ & $89.5(70.5-125.5)$ \\
\hline TBIL (total bilirubin), $\mu \mathrm{mol} / \mathrm{L}$ & $16.7(14.1-24.0)$ \\
\hline Serum AFP level, ng/mL & $52.1(10.6-132.2)$ \\
\hline No. of patients with ICGR-15 > 15\% & $18(32.1)$ \\
\hline No. of patients with prothrombin activity $<75 \%$ & $22(39.2)$ \\
\hline No. of patients with platelet count, $<100 \mathrm{~g} / \mathrm{L}$ & $33(58.9)$ \\
\hline No. of patients with total bilirubin $>21 \mu \mathrm{mol} / \mathrm{L}$ & $15(26.7)$ \\
\hline No. of patients with serum AFP level $>200 \mathrm{ng} / \mathrm{mL}$ & $8(14.2)$ \\
\hline No. of patients with Child-Pugh class A / B & $52(92.9) / 4(7.1)$ \\
\hline \multicolumn{2}{|l|}{ Ascites } \\
\hline Absent & $51(91.1)$ \\
\hline Minor & $5(8.9)$ \\
\hline Esophageal varices & \\
\hline
\end{tabular}




\begin{tabular}{|ll|}
\hline Absent & $50(89.3)$ \\
\hline Grade 1 & $6(10.7)$ \\
\hline Characteristics of tumor diameter & \\
\hline Median value, $\mathrm{mm}$ & $26(18.0-28.0)$ \\
\hline No. of patients with tumor diameter $\leq 20 \mathrm{~mm}$ & $19(33.9)$ \\
\hline No. of patients with tumor diameter $>20 \mathrm{~mm}$ and $\leq 30 \mathrm{~mm}$ & $37(66.1)$ \\
\hline
\end{tabular}

Table 2. Operative characteristics of the NTDP-RFA.

\begin{tabular}{|ll|}
\hline Variables & Date \\
\hline Duration of ablation, min & $8(6-8)$ \\
\hline Length of stay, day & $4(3-5)$ \\
\hline Ablation size, mm & $49.0(44.2-51.0)$ \\
\hline Number of NTDP-RFA session & \\
\hline Complete ablation of single NTDP-RFA session & $55(98.2)$ \\
\hline Two NTDP-RFA sessions & $1(1.8)$ \\
\hline Maximum pain score & $1.55(1.20-1.80)$ \\
\hline postoperative complications & \\
\hline Pleural effusion & $5(8.9)$ \\
\hline Ascites & $2(3.5)$ \\
\hline
\end{tabular}

Table 3. Risk factors for overall tumor progression in 56 patients treated with NTDP-RFA. 


\begin{tabular}{|lllll|}
\hline Variables & Univariable & \multicolumn{3}{l|}{ Multivariable } \\
\cline { 2 - 5 } & HR $(95 \% \mathrm{Cl})$ & $\begin{array}{l}P \\
\text { Value }\end{array}$ & $\mathrm{HR}(95 \% \mathrm{Cl})$ & $\begin{array}{c}P \\
\text { Value }\end{array}$ \\
\hline Age $\geq 60$ y & $0.38(0.08-1.81)$ & 0.228 & & \\
\hline ICGR-15 $>15 \%$ & $2.41(0.69-8.33)$ & $0.164^{*}$ & $2.45(0.57-$ & 0.227 \\
\hline Prothrombin activity $<75 \%$ & $1.59(0.46-5.49)$ & 0.463 & & \\
\hline Platelet count $<100 \mathrm{~g} / \mathrm{L}$ & $1.06(0.30-3.78)$ & 0.918 & \\
\hline Total bilirubin $>21 \mu \mathrm{mol} / \mathrm{L}$ & $0.59(0.12-2.81)$ & 0.516 & & \\
\hline Serum AFP level $>200 \mathrm{ng} / \mathrm{mL}$ & $0.69(0.08-5.49)$ & 0.731 & & \\
\hline $\begin{array}{l}\text { Tumor diameter }>20 \mathrm{~mm} \text { and } \leq 30 \\
\mathrm{~mm}\end{array}$ & $5.26(0.66-$ & $0.115^{\star}$ & $5.34(0.66-$ & \\
\hline
\end{tabular}

\section{Figures}




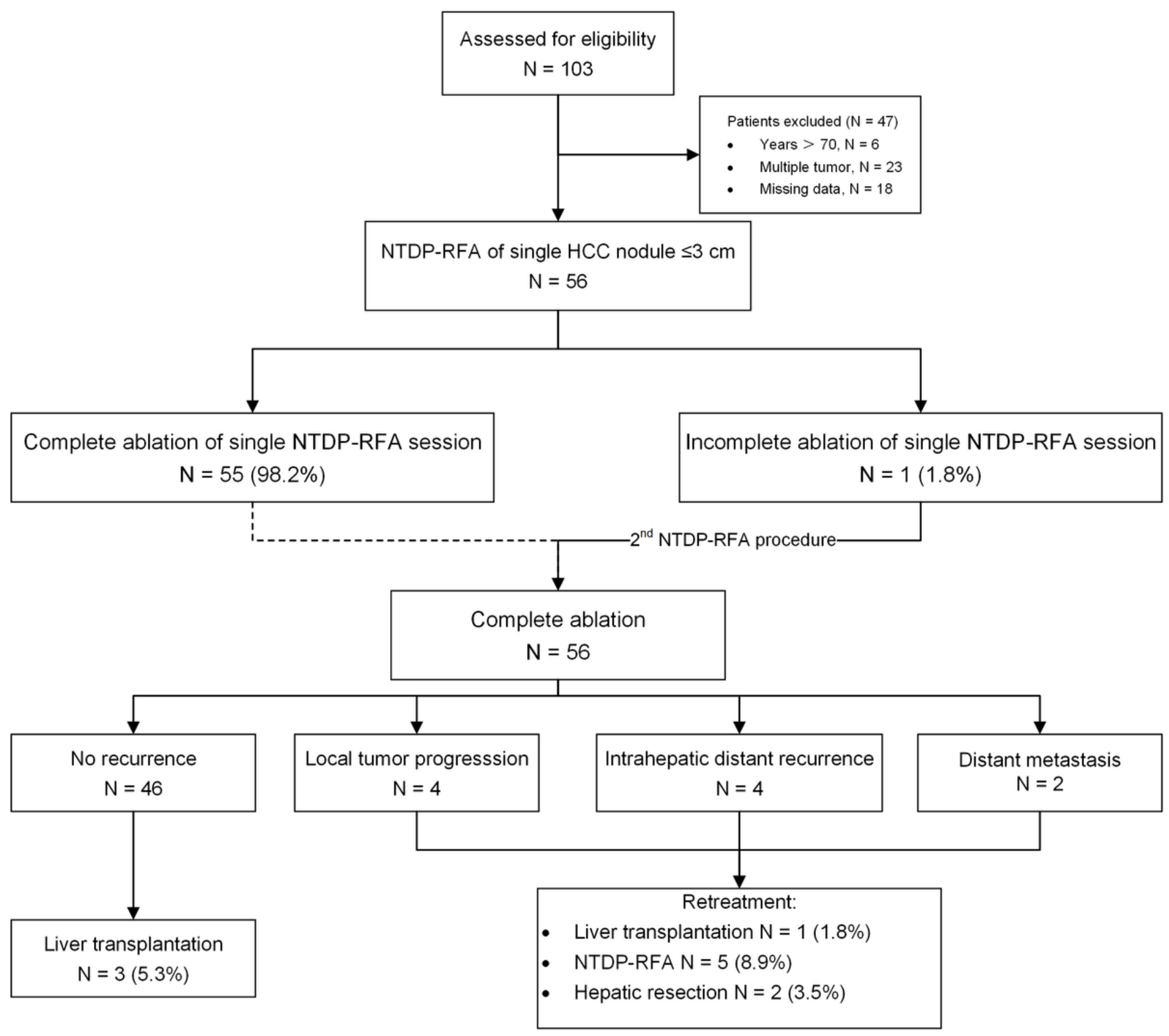

Figure 1

CONSORT showing the outcome of the 56 patients who underwent no-touch combined directed perfusion RFA as a first-line treatment for small HCC with cirrhosis. 


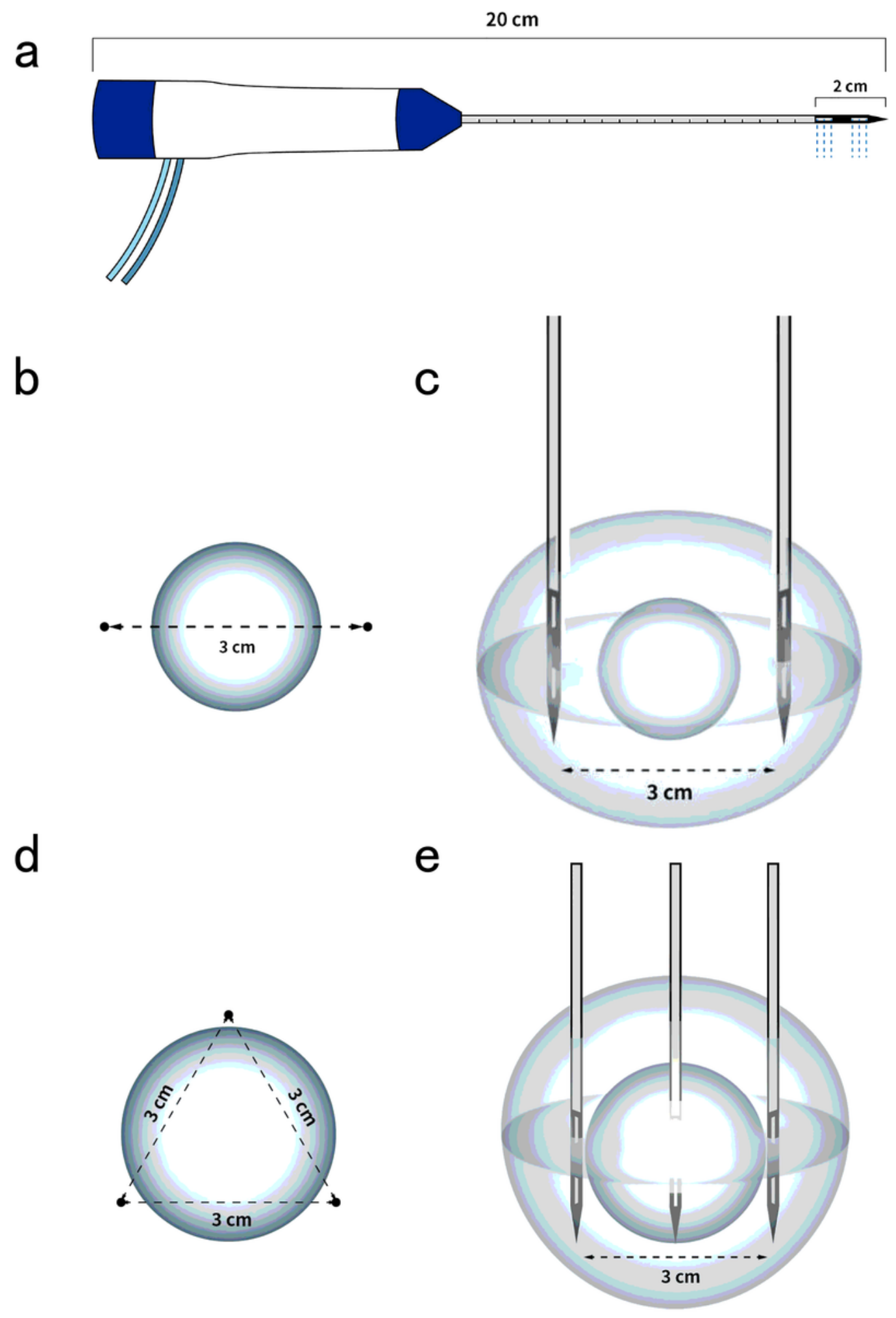

Figure 2

Standardized no-touch combined directed perfusion RFA technique was performed according to the diameter of the tumor. a It shows the specific details of the wet electrode used in no-touch combined directed perfusion RFA procedure. b, c For tumors less than $2 \mathrm{~cm}$ in diameter, two electrodes were inserted parallel into the surrounding non-tumorous liver parenchyma at a distance of $3 \mathrm{~cm}$. d, e For tumors larger 
than $2 \mathrm{~cm}$ and less than $3 \mathrm{~cm}$ in diameter, three electrodes were inserted into the surrounding nontumorous liver parenchyma in an equilateral triangle configuration with side length of $3 \mathrm{~cm}$.
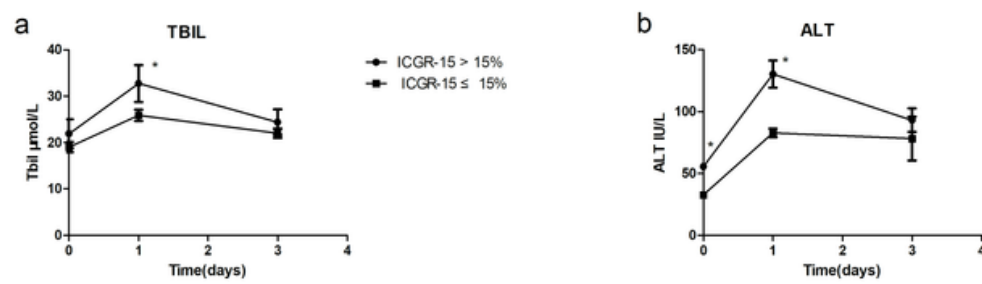

- ICGR $-15>15 \%$
- ICGR $-15 \leq 15 \%$
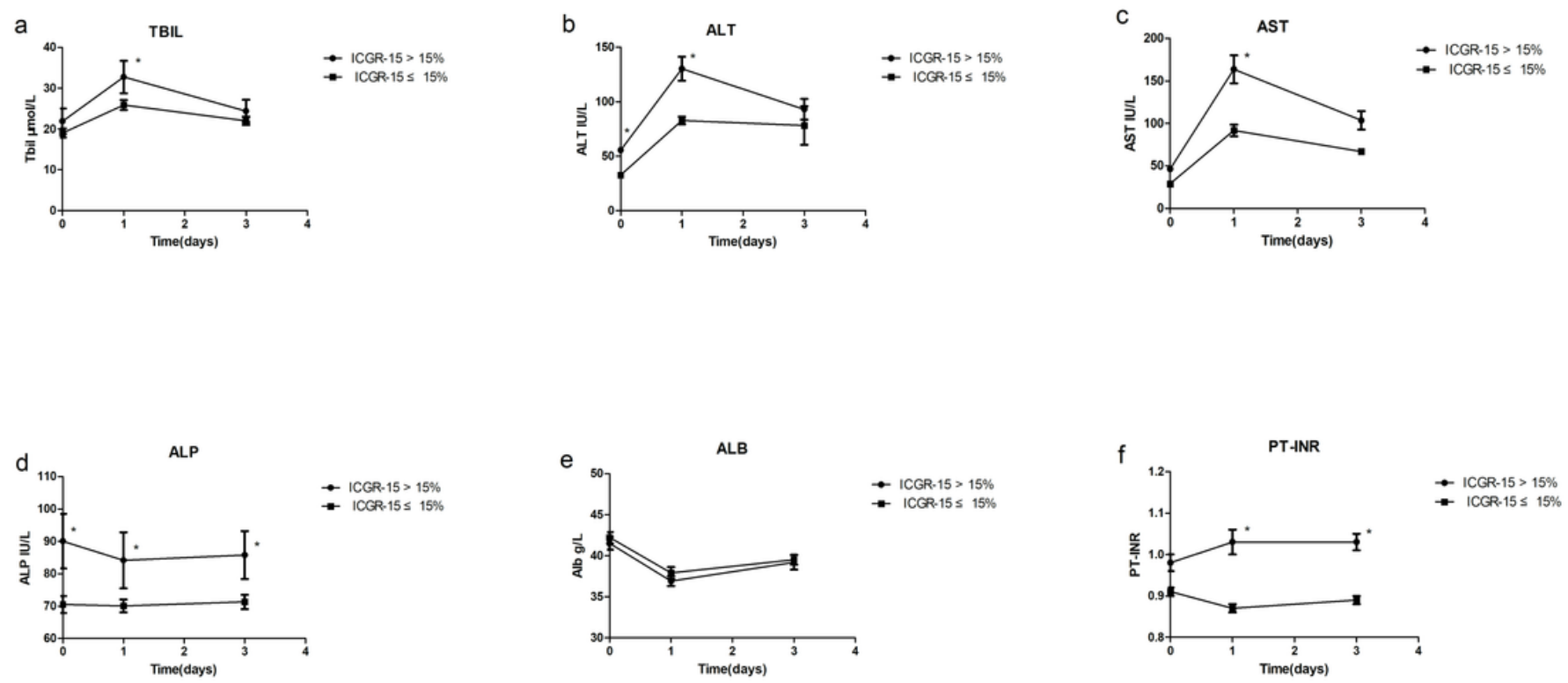

\section{Figure 3}

Serial changes in indicators of liver function. a TBIL (total bilirubin). b ALT (alanine transaminase). c AST ( aspartate aminotransferase). d ALP (alkaline phosphatase). e ALB (albumin). f PT-INR (prothrombin time international normalised ratio). ICGR-15 (indocyanine green retention rate at $15 \mathrm{~min}$ ). ${ }^{*} p<0.050$ versus ICGR-15 $\leq 15 \%$ group (repeated measures ANOVA). The values are expressed as mean with standard error.

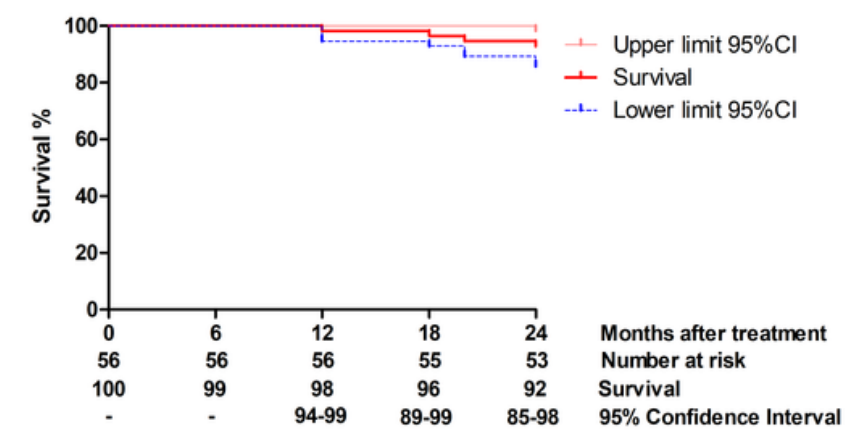

a Local tumor progression-free survival

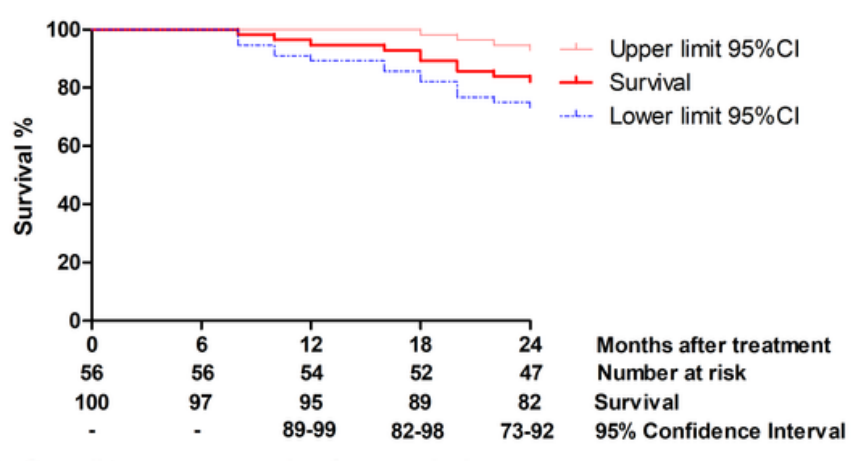

b Overall tumor progression-free survival

\section{Figure 4}


a Local and $\mathrm{b}$ Overall tumor progression-free survival curves in 56 patients treated with no-touch combined directed perfusion RFA. 